\title{
Clinicopathologic features and survival in fibrolamellar carcinoma: comparison with conventional hepatocellular carcinoma with and without cirrhosis
}

Sanjay Kakar ${ }^{1}$, Lawrence J Burgart ${ }^{2}$, Kenneth P Batts ${ }^{3}$, Joaquin Garcia ${ }^{1}$, Dhanpat Jain ${ }^{4}$ and Linda D Ferrell ${ }^{1}$

${ }^{1}$ Department of Anatomic Pathology, UCSF/VA Medical Center, San Francisco, CA, USA; ${ }^{2}$ Mayo Clinic, Rochester, MN, USA; ${ }^{3}$ Abbott Northwestern Hospital, Minneapolis, MN, USA and ${ }^{4}$ Yale University School of Medicine, New Haven, CT, USA

\begin{abstract}
Fibrolamellar carcinoma arises in noncirrhotic livers of young individuals and has been considered to be less aggressive than conventional hepatocellular carcinoma. This study compares survival and clinicopathologic features of fibrolamellar carcinoma with hepatocellular carcinoma arising in noncirrhotic and cirrhotic livers. Clinical and pathologic features including age, gender, tumor size, stage and survival were recorded in 20 resected cases of fibrolamellar carcinoma. Survival was compared with resected hepatocellular carcinoma without $(n=32)$ and with cirrhosis $(n=30)$. Proliferative activity was determined by immunohistochemistry for Ki-67. In all, $12(60 \%)$ patients with fibrolamellar carcinoma died during follow-up; the 5-year survival was $45 \%$. Mortality in fibrolamellar carcinoma was higher with metastatic disease at presentation $(6 / 7,86 \% v s 5 / 13,39 \%$, $P=0.06)$. Age, gender and tumor size did not correlate with survival. The 5 -year $(45$ vs $56 \%, P=0.4)$ as well as overall survival (40 vs $56.3 \%, P=0.3$ ) was similar in fibrolamellar carcinoma and hepatocellular carcinoma without cirrhosis. The 5-year and overall survival in hepatocellular carcinoma with cirrhosis was 27 and $23.3 \%$, respectively, which was not significantly different compared to fibrolamellar carcinoma $(P=0.2)$. Among the cases without metastases at presentation, 5 -year survival in fibrolamellar carcinoma (62\%) and hepatocellular carcinoma without cirrhosis $(57 \%)$ was significantly better $(P=0.03)$ than hepatocellular carcinoma with cirrhosis $(27 \%)$. The mean $\mathrm{Ki}-67$ index was similar in all three groups $(P=0.1)$. In conclusion, fibrolamellar carcinoma is an aggressive neoplasm with $45 \% 5$-year survival and overall mortality of $60 \%$. Nearly half the patients develop lymph node or distant metastasis. The prognosis of fibrolamellar carcinoma is similar to conventional hepatocellular carcinoma. Among nonmetastatic cases, the prognosis is better in fibrolamellar carcinoma and hepatocellular carcinoma without cirrhosis compared to hepatocellular carcinoma with cirrhosis. The better outcome in fibrolamellar carcinoma appears to be due to the absence of cirrhosis rather than its distinct clinicopathologic features.
\end{abstract}

Modern Pathology (2005) 18, 1417-1423. doi:10.1038/modpathol.3800449; published online 27 May 2005

Keywords: fibrolamellar; hepatocellular carcinoma; survival; cirrhosis

Fibrolamellar hepatocellular carcinoma is a variant of hepatocellular carcinoma with distinct clinicopathologic features. It occurs at a young age ${ }^{1-3}$ and lacks common risk factors for hepatocellular carcinoma like viral hepatitis and cirrhosis. ${ }^{4-6}$ Elevated alpha fetoprotein (AFP) levels are infrequent. ${ }^{2,6}$

Correspondence: Dr S Kakar, MD, Department of Anatomic Pathology, UCSF/VA Medical Center, San Francisco, CA 94121, USA.

E-mail: skakar@itsa.ucsf.edu

Received 9 March 2005; revised 18 April 2005; accepted 19 April 2005; published online 27 May 2005
The defining feature of fibrolamellar carcinoma is its triad of histologic characteristics, viz. tumor cells with deeply eosinophilic cytoplasm, presence of macronucleoli and abundant fibrous stroma arranged in thin parallel lamellae around the tumor cells. . $^{1,4,5,7}$

Many studies have advocated that fibrolamellar carcinoma is less aggressive than conventional hepatocellular carcinoma. ${ }^{1,3-5,8-14}$ Several pathology and hepatology texts mention that fibrolamellar carcinoma is associated with a favorable prognosis. ${ }^{15-18}$ However, other studies have failed to confirm the observation of a better outcome in 
fibrolamellar carcinoma. ${ }^{2,6,19,20,21}$ The World Health Organization blue book lists fibrolamellar carcinoma as a distinct variant of hepatocellular carcinoma, but refrains from any comment about its outcome compared to conventional hepatocellular carcinoma. ${ }^{22}$ In our experience, fibrolamellar carcinoma is an aggressive tumor and we have observed recurrence and metastasis in many cases of fibrolamellar carcinoma after initial resection.

Cirrhosis is a well-established poor prognostic factor in hepatocellular carcinoma. ${ }^{23,24}$ Since fibrolamellar carcinoma almost always arises in noncirrhotic liver, the apparent better outcome in fibrolamellar carcinoma may be related to lack of cirrhosis. Approximately $20 \%$ of conventional hepatocellular carcinoma arise in the absence of cirrhosis. ${ }^{25}$ The survival in fibrolamellar carcinoma cases should be compared to conventional hepatocellular carcinoma arising in noncirrhotic liver to avoid the confounding factor of cirrhosis. There have been few studies that have simultaneously compared fibrolamellar carcinoma with hepatocellular carcinoma with and without cirrhosis. ${ }^{6,12,19}$ The goal of this study was to compare the survival and clinicopathologic features of fibrolamellar carcinoma with hepatocellular carcinoma arising in noncirrhotic and cirrhotic livers.

\section{Materials and methods}

The study population comprised of 20 patients with resected fibrolamellar carcinoma and 62 patients with conventional hepatocellular carcinoma (30 arising in cirrhotic liver and 32 in noncirrhotic liver). The cases were retrieved from UCSF Medical Center, Mayo Clinic and Yale New Haven Hospital by searching for 'fibrolamellar carcinoma' in the diagnosis line of liver resection specimens from 1987 to 2000. The slides were reviewed to confirm the diagnosis. Four cases originally diagnosed as fibrolamellar carcinoma lacked the typical histological features and were reclassified as scirrhous hepatocellular carcinoma. These cases are not included in the study group of 20 cases. All tumors classified as fibrolamellar carcinoma showed abundant granular cytoplasm, enlarged nucleus with a prominent nucleolus and lamellar plate-like fibrosis (Figure 1). Clinical and pathologic features including age, gender, tumor size, stage and survival data were recorded. Among the hepatocellular carcinoma cases arising in cirrhotic liver, the etiology of cirrhosis was hepatitis B $(n=7)$, hepatitis C $(n=11)$, hereditary hemochromatosis $(n=1)$ and cryptogenic $(n=11)$. Additional histologic features were recorded for fibrolamellar carcinoma cases including presence of bile, cytoplasmic hyaline globules and pale bodies. Rhodanine stain for copper was performed in 12 cases of fibrolamellar carcinoma. Positive results were scored as mild, moderate or marked. The clinicopathologic features

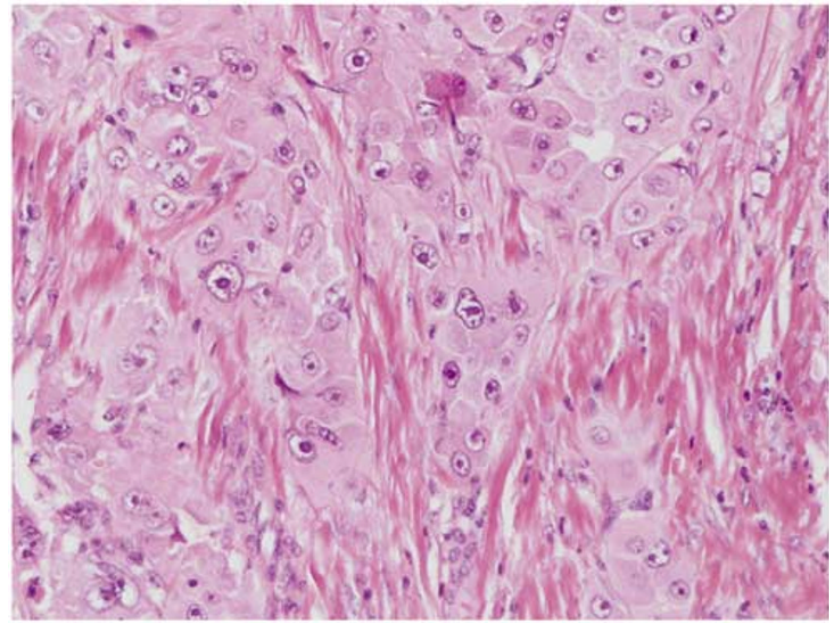

Figure 1 Fibrolamellar carcinoma characterized by lamellar pattern of fibrosis, oncocytic cytoplasm and prominent eosinophilic nucleoli.

and survival in fibrolamellar carcinoma was compared with hepatocellular carcinoma arising in noncirrhotic and cirrhotic liver.

The fibrolamellar carcinoma cases were examined by paraffin immunohistochemistry using rabbit polyclonal antibody against AFP (1:500, Dako, Carpinteria, CA, USA) and mouse monoclonal antibodies against chromogranin (1:75, Boehringer Mannheim, Petersburg, VA, USA) and synaptophysin (1:40, MP Biomedicals, Irvine, CA, USA). Proliferation activity in both fibrolamellar carcinoma and hepatocellular carcinoma was determined by using mouse monoclonal antibody against Ki-67 (1:100, Dako, Carpinteria, CA, USA). Deparaffinized slides were microwaved in $10 \mathrm{mM}$ citrate buffer for $10 \mathrm{~min}$ for antigen retrieval. The endogenous peroxidase activity was blocked by $3 \% \mathrm{H}_{2} \mathrm{O}_{2}$. The sections were incubated overnight with the primary antibody at $4^{\circ} \mathrm{C}$ Biotinylated horse anti-mouse antibody (Vector Labs, Burlingame, CA, USA) was used as the secondary antibody and diacetyl benzidine was used for color development. The Ki-67 index was determined by counting the number of positive cells in five high-power fields and expressing the result as a percentage. Fisher's exact test and $\chi^{2}$ test were used for statistical analysis. Log-rank test and Kaplan-Meier curves were used for survival analysis.

\section{Results}

\section{Fibrolamellar Carcinoma}

The mean age based on 19 cases of fibrolamellar carcinoma (age not known in one case) was 27 years (range 16-47 years, 11 male, nine female). In all, 16 $(84 \%)$ cases were below 40 years of age. Multiple primary tumors were present in two cases. Serum AFP was elevated in $3 / 13(23 \%)$ patients; levels 
Table 1 Clinicopathologic features and survival in fibrolamellar carcinoma (figures in parenthesis reflect percentages)

\begin{tabular}{|c|c|c|c|}
\hline & \multicolumn{2}{|c|}{ Survival } & \multirow[t]{2}{*}{$\mathrm{P}$-valu } \\
\hline & $<5$ years & $\geq 5$ years & \\
\hline \multicolumn{4}{|c|}{ Age (years) } \\
\hline$<40$ & $9(56)$ & $7(44)$ & 0.6 \\
\hline$\geq 40$ & $2(67)$ & $1(33)$ & \\
\hline \multicolumn{4}{|l|}{ Gender } \\
\hline Female & $5(50)$ & $5(50)$ & 0.4 \\
\hline Male & $3(33)$ & $6(67)$ & \\
\hline \multicolumn{4}{|c|}{ Tumor size $(\mathrm{cm})$} \\
\hline$<10$ & $2(40)$ & $3(60)$ & 0.2 \\
\hline$\geq 10$ & $8(73)$ & $3(27)$ & \\
\hline \multicolumn{4}{|c|}{ Metastasis at presentation } \\
\hline Absent & 5 (38) & $8(62)$ & 0.06 \\
\hline Present & $6(86)$ & $1(14)$ & \\
\hline
\end{tabular}

were not known in the other cases. The levels were $<100 \mathrm{ng} / \mathrm{ml}$ in two cases and $612 \mathrm{ng} / \mathrm{ml}$ in one case. Stainable copper was present in $9 / 12(75 \%)$ cases examined. The staining was mild in six cases and marked in three cases. AFP was negative by immunohistochemistry in all 14 cases examined. Immunohistochemistry for synaptophysin and chromogranin was negative in all 12 cases tested.

A total of $12(60 \%)$ patients died of the disease; the 5 -year survival was $45 \%$. Seven of the $20(35 \%)$ patients had lymph node metastasis at presentation. The 5-year survival was higher in patients with localized disease at initial presentation $(8 / 13,62 \%$ vs $1 / 7,14 \%, P=0.06)$. Age, gender and tumor size did not correlate with survival (Table 1).

\section{Comparison with Conventional Hepatocellular Carcinoma}

Fibrolamellar carcinoma occurred at a younger age and was associated with a larger tumor size compared to HCC (Table 2). Cytoplasmic pale bodies were identified in eight $(40 \%)$ fibrolamellar carcinoma cases, but were not seen in hepatocellular carcinoma (Figure 2). Survival analysis among all cases irrespective of stage showed that the 5-year (45 vs $56 \%, P=0.4$ ) as well as overall survival (40 vs $56 \%, P=0.3$ ) was similar in fibrolamellar carcinoma and hepatocellular carcinoma without cirrhosis. The 5-year and overall survival in hepatocellular carcinoma with cirrhosis was 27 and $23 \%$, respectively, which was worse compared to fibrolamellar carcinoma (Figure 3), but the difference was not statistically significant $(P=0.2)$.

When survival analysis was performed in cases with same-stage disease by examining tumors localized to the liver at presentation (Table 1, Figure
Table 2 Survival and clinicopathologic features in fibrolamellar carcinoma and hepatocellular carcinoma (HCC) with and without cirrhosis

\begin{tabular}{|c|c|c|c|c|}
\hline & $\begin{array}{c}\text { Fibrolamellar } \\
\text { carcinoma } \\
(\mathrm{n}=20)\end{array}$ & $\begin{array}{c}\text { HCC } \\
\text { without } \\
\text { cirrhosis } \\
(\mathrm{n}=32)\end{array}$ & $\begin{array}{l}\text { HCC with } \\
\text { cirrhosis } \\
(\mathrm{n}=30)\end{array}$ & $\mathrm{P}$-value \\
\hline \multicolumn{5}{|l|}{ Age (years) ${ }^{\mathrm{b}}$} \\
\hline$<40$ & $16(84)$ & $3(9)$ & 4 (13) & $<0.001$ \\
\hline$\geq 40$ & $3(16)$ & $29(91)$ & $26(87)$ & \\
\hline \multicolumn{5}{|l|}{ Gender ${ }^{b}$} \\
\hline Female & $9(47)$ & $14(44)$ & $25(83)$ & 0.2 \\
\hline Male & $10(53)$ & $18(56)$ & $5(17)$ & \\
\hline \multicolumn{5}{|l|}{ Tumor size $(\mathrm{cm})^{\mathrm{b}}$} \\
\hline$<10$ & $5(31)$ & $19(59)$ & $26(87)$ & 0.003 \\
\hline$\geq 10$ & $11(69)$ & $13(41)$ & $4(13)$ & \\
\hline 'Pale bodies' & $8(40)$ & 0 & 0 & $<0.001$ \\
\hline $\begin{array}{l}\text { 5-year survival- } \\
\text { nonmetastatic } \\
\text { cases }\end{array}$ & $62 \%$ & $58 \%$ & $27 \%$ & 0.03 \\
\hline
\end{tabular}

${ }^{\mathrm{a}}$ For age, gender and size, $P$-values reflect comparison of fibrolamellar carcinoma with conventional hepatocellular carcinoma (with and without cirrhosis). For survival, $P$-value reflects comparison of fibrolamellar carcinoma and hepatocellular carcinoma with cirrhosis ( $P$-values for hepatocellular carcinoma without cirrhosis vs hepatocellular carcinoma with cirrhosis were also the same). Numbers within parenthesis reflect percentages.

${ }^{\mathrm{b}}$ Numbers in some fibrolamellar carcinoma columns do not add to 20 as information was not available in all cases.

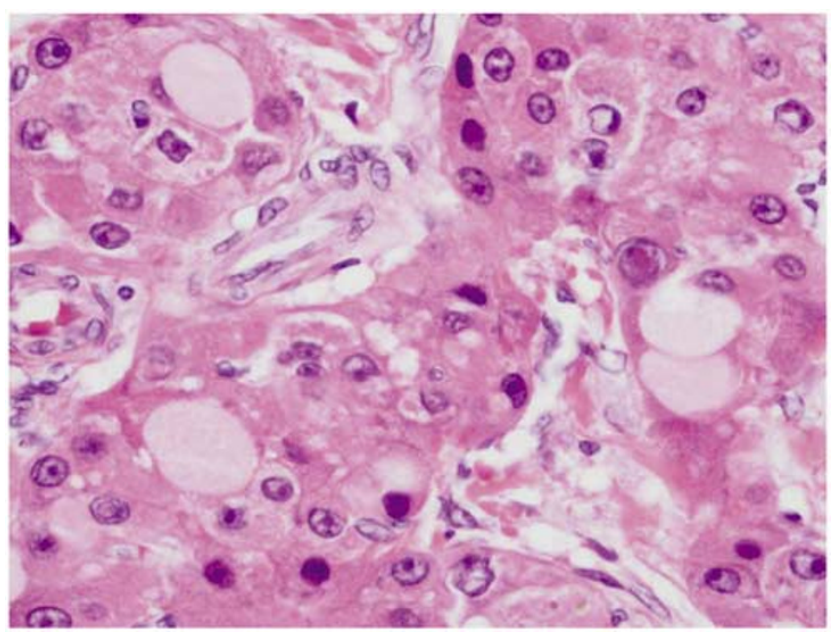

Figure 2 Fibrolamellar carcinoma showing intracytoplasmic pale bodies in tumor cells.

4), the 5-year survival in fibrolamellar carcinoma $(62 \%)$ and hepatocellular carcinoma without cirrhosis $(58 \%)$ was similar, but survival in both groups was significantly better than hepatocellular carcinoma with cirrhosis $(27 \%)$.

The mean Ki-67 index was 5.0 (range 0.2-17) in fibrolamellar carcinoma, 7.8 (range 0.1-27) in hepatocellular carcinoma without cirrhosis and 9.5 (range 0.5-26) in hepatocellular carcinoma with 


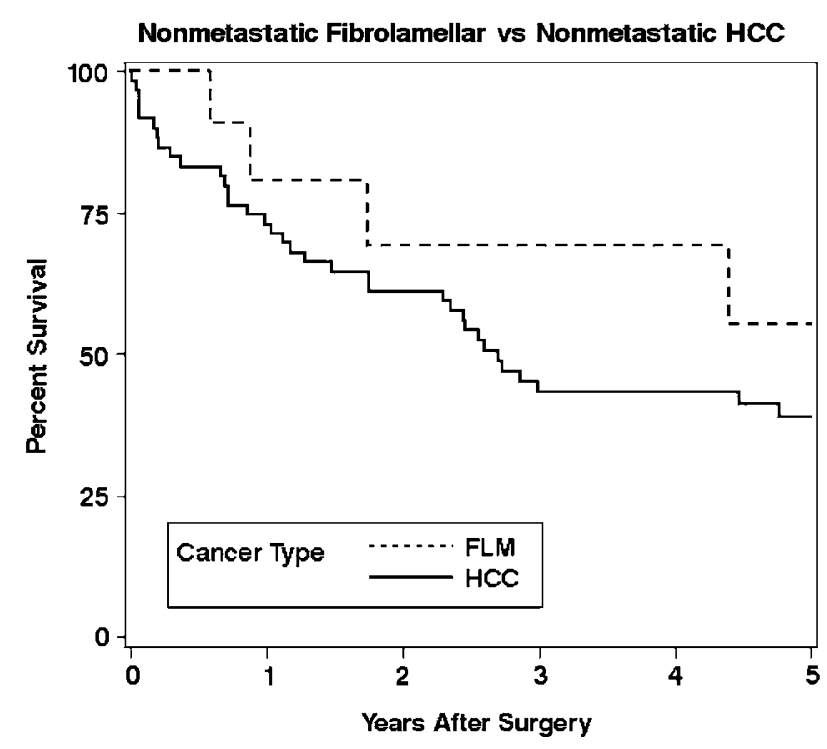

FLM- Fibrolamellar carcinoma HCC- Hepatocellular carcinoma

Figure 3 The 5-year survival in same-stage fibrolamellar carcinoma (confined to the liver at presentation) is similar to conventional hepatocellular carcinoma $(P=0.4)$

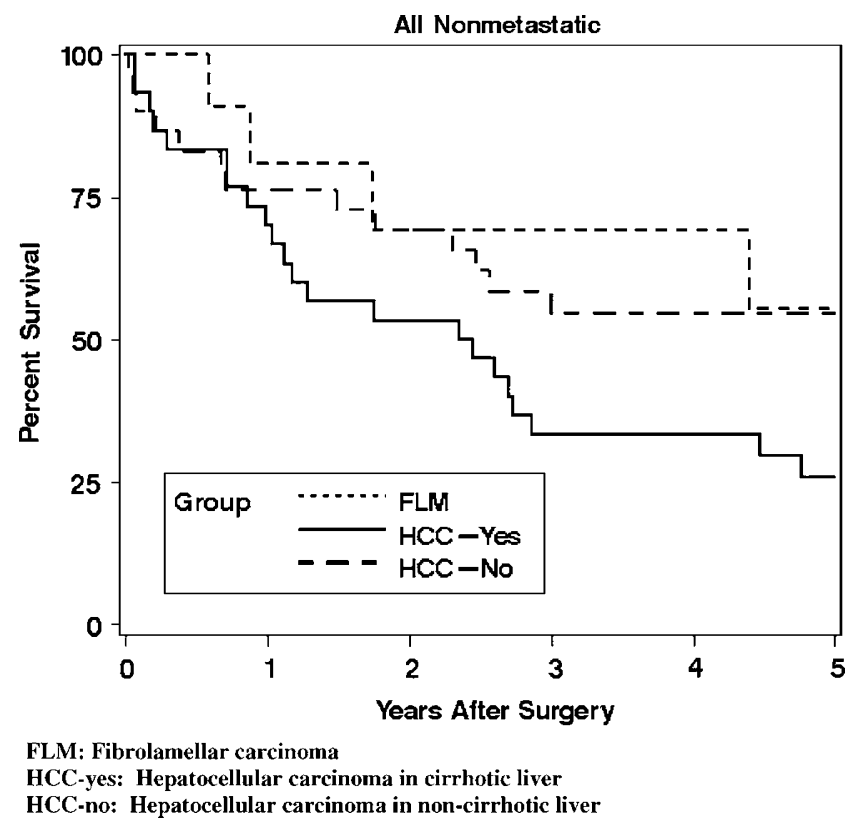

Figure 4 Survival in same-stage fibrolamellar carcinoma (confined to the liver at presentation) is similar to hepatocellular carcinoma without cirrhosis $(P=0.4)$ and better than hepatocellular carcinoma with cirrhosis $(P=0.03)$.

cirrhosis $(P=0.1)$. The Ki-67 index did not correlate with survival.

\section{Discussion}

Fibrolamellar carcinoma is an uncommon variant of hepatocellular carcinoma with distinctive morpho- logy and clinical setting. It has been widely regarded that fibrolamellar carcinoma has relatively favorable natural history and is associated with better outcome compared to conventional hepatocellular carcinoma.

This study confirms the distinctive clinicopathologic features of fibrolamellar carcinoma as reported in literature. The tumors in fibrolamellar carcinoma cases were significantly larger than conventional hepatocellular carcinoma; similar findings have been reported in literature. ${ }^{5,9,11}$ Majority of the tumors occurred in patients less than 40 years old, with only $15 \%$ above the age of 40 years. A review of some large series reveals that $80 \%$ of fibrolamellar carcinoma patients present before 35 years and only $11 \%$ after the age of 40 years. It is important to strictly use established criteria for the diagnosis of fibrolamellar carcinoma, especially in older individuals. In ambiguous cases, the presence of 'pale bodies' would strongly favor fibrolamellar carcinoma, although they have been described in conventional hepatocellular carcinoma, especially the scirrhous variant. ${ }^{22}$ Stainable copper was found in $75 \%$ of fibrolamellar carcinoma in our series. The presence of copper and copper-binding protein was cited as a specific feature of fibrolamellar carcinoma, ${ }^{26,27}$ but was later described in conventional HCC as well. ${ }^{28}$ Serum AFP was elevated in 3/18 (16.7\%) cases, although levels were less than $70 \mathrm{ng} / \mathrm{ml}$ except in one case. A prior review noted elevated AFP in $7 \%$ of fibrolamellar carcinoma compared to $83 \%$ of conventional hepatocellular carcinoma. ${ }^{2}$ Synaptophysin and chromogranin were negative in all our cases. Nonspecific enolase and neurotensin have been reported to be positive in fibrolamellar carcinoma. ${ }^{29,30}$ Structures resembling neurosecretory granules have been noticed in some ${ }^{29}$ but not all ultrastructural studies. ${ }^{1,31}$ The significance of this 'neuroendocrine differentiation' remains unclear, but is unlikely to be helpful in distinction from conventional HCC as up to one-fourth of the latter may also show 'neuroendocrine differentiation'. ${ }^{30,32,33}$ Immunohistochemistry for AFP was negative in all cases tested, similar to other studies, ${ }^{8}$ although focal positive reaction in small groups of cells has been reported. ${ }^{31}$

Several studies have emphasized the better survival in fibrolamellar carcinoma compared to conventional hepatocellular carcinoma. ${ }^{1,3-5,8-14}$ However, these results could not be confirmed by many authors. ${ }^{2,6,19,20}$ The present study provides further evidence that fibrolamellar carcinoma is an aggressive neoplasm associated with 5-year survival of less than $50 \%$. Although the majority of cases occurred in patients less than 40 years old, nearly $40 \%$ of patients died even when fibrolamellar carcinoma was localized to the liver at presentation. The outcome was particularly dismal (5-year survival $\sim 15 \%$ ) in patients with metastatic disease at presentation. The 5-year survival in resected fibrolamellar carcinoma in various reports ranges from 
Table 3 Summary of results obtained in some of the larger series of fibrolamellar carcinoma (FLM)

\begin{tabular}{|c|c|c|c|}
\hline Study (reference number) & $\begin{array}{l}\text { FLM-case } \\
\text { number }\end{array}$ & Survival & Comments \\
\hline Wood et al ${ }^{12}$ & 15 & $\begin{array}{l}5 \text {-year survival } 45 \% \text { in resected cases; overall } \\
\text { survival } 25 \text { vs } 0 \% \text { in HCC }\end{array}$ & $\begin{array}{l}\text { HCC in noncirrhotic liver not } \\
\text { separately analyzed }\end{array}$ \\
\hline Starzl et $a l^{10}$ & 14 & $12 / 14$ alive (variable follow-up) & $\begin{array}{l}\text { 5-year follow-up in only } 2 / 12 \text { surviving } \\
\text { patients }\end{array}$ \\
\hline Craig et $a l^{1}$ & 23 & $\begin{array}{l}\text { Overall survival } 30 \% \text {, considered better than } \\
\text { HCC }\end{array}$ & $\begin{array}{l}\text { HCC in noncirrhotic liver not } \\
\text { separately analyzed; four FLM cases in } \\
\text { cirrhotic/fibrotic liver }\end{array}$ \\
\hline Berman et $a l^{5}$ & 12 & 5-year survival $68 \%$, considered better than HCC & $\begin{array}{l}\text { HCC in noncirrhotic liver not } \\
\text { separately analyzed; three cases had } \\
\text { admixed areas of typical HCC }\end{array}$ \\
\hline Soreide et $a l^{11}$ & 9 & All six resected cases alive & None of the cases had 5-year follow-up \\
\hline Farhi et $a l^{9}$ & 10 & $50 \%$ overall survival; none in HCC & $\begin{array}{l}\text { HCC in noncirrhotic liver not } \\
\text { separately analyzed }\end{array}$ \\
\hline Lack et $a l^{8}$ & 5 & $\begin{array}{l}\text { Two of three resected cases died; overall } 80 \% \\
\text { patients died within } 3 \text { years; mean survival better } \\
\text { than HCC }\end{array}$ & $\begin{array}{l}\text { HCC in noncirrhotic liver not } \\
\text { separately analyzed }\end{array}$ \\
\hline Pinna et $a l^{14}$ & 28 & 5 -year survival $75 \%$ & $\begin{array}{l}\text { Comparison with same stage HCC not } \\
\text { shown; FLM cases not reviewed and } \\
\text { diagnostic criteria not specified }\end{array}$ \\
\hline El Serag and Davila ${ }^{3}$ & 68 & 5-year survival $32 \%$ compared to $7 \%$ in HCC & $\begin{array}{l}\text { HCC in noncirrhotic liver not } \\
\text { separately analyzed }\end{array}$ \\
\hline Nagorney et al $1^{19}$ & 16 & $\begin{array}{l}\text { Overall survival } 31 \% \text {, better than HCC in } \\
\text { noncirrhotic liver }\end{array}$ & $\begin{array}{l}\text { No difference in survival between FLM } \\
\text { and HCC in noncirrhotic liver among } \\
\text { resected cases }\end{array}$ \\
\hline Ringe et $a l^{6}$ & 20 & $\begin{array}{l}5 \text {-year survival } 43 \% \text { in resected cases; } 37 \% \text { in all } \\
\text { cases }\end{array}$ & Outcome in FLM similar to HCC \\
\hline Haas et $a l^{20}$ & 14 & 5-year survival $<25 \%$ in both FLM and HCC & Outcome in FLM similar to HCC \\
\hline Katzenstein et $a l^{21}$ & 10 & 5 -year survival $30 \%$; overall mortality $60 \%$ & Outcome in FLM similar to HCC \\
\hline Present & 20 & $\begin{array}{l}\text { 5-year survival } 45 \% \text {; overall } 62 \% \text { in stage I } \\
\text { disease }\end{array}$ & $\begin{array}{l}\text { Outcome in FLM similar to same-stage } \\
\text { HCC in noncirrhotic liver; better than } \\
\text { HCC in cirrhotic liver }\end{array}$ \\
\hline
\end{tabular}

$<25$ to $75 \%{ }^{1,4,5,6,8,9,11,12,14,18,19,21}$ The aggressive nature of fibrolamellar carcinoma is apparent from the fact that in several studies in which fibrolamellar carcinoma had a better outcome compared to conventional hepatocellular carcinoma, the 5-year survival was only $20-40 \% .^{1,8,12}$ Ploidy studies have shown that all fibrolamellar carcinoma cases are aneuploid or polyploid, which is in keeping with an aggressive tumor. ${ }^{34}$ Similarly, chromosomal genomic hybridization has shown chromosomal gains and losses that are similar to conventional hepatocellular carcinoma. ${ }^{35}$

Several reasons could explain the discrepant results reported in literature. Fibrolamellar carcinoma is a rare tumor and majority of the reported series are small (Table 3). This may lead to lack of sufficient power for statistical analysis. Another reason may be the criteria used for the diagnosis of fibrolamellar carcinoma. It has been advocated that a triad of histological features be used for diagnosisabundant eosinophilic cytoplasm, plate-like lamellar fibrosis and large nuclei with marginalized chromatin and prominent nucleolus. ${ }^{7}$ Reliance on two features may lead to erroneous diagnosis of some hepatocellular carcinoma variants (like scirrhous variant and hepatocellular carcinoma with oncocytic cytoplasm) as fibrolamellar carcinoma. Many studies have not used this diagnostic triad to identify cases of fibrolamellar carcinoma; in some series, the diagnostic criteria are not clearly outlined $^{12}$ or the original histological diagnosis was not reviewed before being included in the study. ${ }^{3}$ Some studies have included cases that occurred in cirrhotic liver and are unlikely to be fibrolamellar carcinoma. ${ }^{1}$ In some series, tumors with fibrolamellar carcinoma-like areas were included even though other areas showed features typical of conventional hepatocellular carcinoma. ${ }^{5}$ In our experience, the clinicopathologic features of these tumors like advanced age and background of cirrhosis resemble conventional hepatocellular carcinoma rather than fibrolamellar carcinoma (unpublished observations). These 'mixed' tumors have also been reported in association with hepatitis $\mathrm{C}$ and cirrhosis, which supports the view that they should be classified as conventional hepatocellular carcinoma. ${ }^{36}$ In some studies, lack of adequate follow-up may contribute to the conclusion of better prognosis in fibrolamellar carcinoma. In a series reported by Starzl et al, ${ }^{10} 12 / 14$ fibrolamellar carcinoma patients were alive after transplantation or resection. However, 5-year follow-up was available in only two of these 12 patients.

Perhaps the most important reason for the varying results in literature is that fibrolamellar carcinoma has been compared to hepatocellular carcinoma as a 
whole. Hepatocellular carcinoma is not a prognostically homogeneous entity but can be divided into several subgroups, which may have distinct biological behavior. Examples of these subgroups include hepatocellular carcinoma arising in noncirrhotic liver, small encapsulated hepatocellular carcinoma and well-differentiated hepatocellular carcinoma. However, the overwhelming majority of hepatocellular carcinoma $(>80 \%)$ arise in cirrhotic liver. ${ }^{25}$ Cirrhosis is a well-known adverse prognostic feature in hepatocellular carcinoma. ${ }^{23,24}$ Hence, when survival in fibrolamellar carcinoma is compared to hepatocellular carcinoma as a whole, the poor outcome in the latter group as a result of cirrhosis is likely to skew the results. In a vast majority of studies, fibrolamellar carcinoma has been compared to hepatocellular carcinoma as a whole (Table 3). The largest and most recent population-based study of 68 cases of fibrolamellar carcinoma using information collected from the Surveillance, Epidemiology and End Results (SEER) program showed superior survival in fibrolamellar carcinoma, but compared it to hepatocellular carcinoma as a group without separately analyzing hepatocellular carcinoma with and without cirrhosis. ${ }^{3}$ The study also relies on the original pathology diagnosis and cases were not reviewed to confirm the diagnosis of fibrolamellar carcinoma before inclusion in the study.

There are few studies in literature that have compared fibrolamellar carcinoma to hepatocellular carcinoma by separating the latter cases into those arising in noncirrhotic liver and cirrhotic liver. Nagorney et $a l^{19}$ reported no significant difference in survival in 12 cases of resected fibrolamellar carcinoma compared to hepatocellular carcinoma in noncirrhotic liver. Ruffin in a series of five fibrolamellar carcinoma case reports suggested that the differences between fibrolamellar carcinoma and comparable subgroups of hepatocellular carcinoma are less distinct. $^{2}$ In a study of 20 cases of fibrolamellar carcinoma, Ringe et $a l^{6}$ reported a 5year survival of $37 \%$ and did not find a better prognosis compared to conventional hepatocellular carcinoma. Our study supports these findings and fails to confirm the assertion that fibrolamellar carcinoma has a favorable outcome. We found that survival was similar in resected fibrolamellar carcinoma and hepatocellular carcinoma in noncirrhotic liver among the cases confined to the liver at presentation. The survival in both these groups was significantly better than hepatocellular carcinoma arising in cirrhotic liver. It has been argued that better survival in fibrolamellar carcinoma may be related to higher resectability rate. ${ }^{3,19}$ Since our analysis includes only resected cases of fibrolamellar carcinoma and hepatocellular carcinoma, this issue cannot be addressed by our study.

In conclusion, our results indicate that fibrolamellar carcinoma is an aggressive tumor associated with less than $50 \%$ 5-year survival even in resect- able cases. When same-stage disease is considered, the survival in fibrolamellar carcinoma is similar to hepatocellular carcinoma arising in noncirrhotic liver, and better than hepatocellular carcinoma arising in cirrhotic liver. The better survival in fibrolamellar carcinoma is likely to be related to the absence of cirrhosis rather than the unique clinicopathologic features of the tumor.

\section{Acknowledgements}

We thank the UCSF Liver Center (P30 DK26743) for providing assistance with sample processing for immunohistochemistry.

\section{References}

1 Craig JR, Peters RL, Edmondson HA, et al. Fibrolamellar carcinoma of the liver: a tumor of adolescents and young adults with distinctive clinico-pathologic features. Cancer 1980;46:372-379.

2 Ruffin IV MT. Fibrolamellar hepatoma. Am J Gastroenterol 1990;85:577-581.

3 El-Serag HB, Davila JA. Is fibrolamellar carcinoma different from hepatocellular carcinoma? A US population-based study. Hepatology 2004;39:798-803.

4 Hodgson HJ. Fibrolamellar cancer of the liver. J Hepatol 1987;5:241-247.

5 Berman MA, Burnham JA, Sheahan DG. Fibrolamellar carcinoma of the liver: an immunohistochemical study of nineteen cases and a review of the literature. Hum Pathol 1988;19:784-794.

6 Ringe B, Wittekind C, Weimann A, et al. Results of hepatic resection and transplantation for fibrolamellar carcinoma. Surg Gynecol Obstet 1992;175:299-305.

7 Burgart LJ, Martinez CJM, Batts KP. Fibrolamellar hepatoma-importance of using a strict definition (abstract). Mod Pathol 1994;7:129A.

8 Lack EE, Neave C, Vawter GF. Hepatocellular carcinoma. Review of 32 cases in childhood and adolescence. Cancer 1983;52:1510-1515.

9 Farhi DC, Shikes RH, Murari PJ, et al. Hepatocellular carcinoma in young people. Cancer 1983;52:15161525.

10 Starzl TE, Iwatsuki S, Shaw Jr BW, et al. Treatment of fibrolamellar hepatoma with partial or total hepatectomy and transplantation of the liver. Surg Gynecol Obstet 1986;162:145-148.

11 Soreide O, Czerniak A, Bradpiece H, et al. Characteristics of fibrolamellar hepatocellular carcinoma. A study of nine cases and a review of the literature. Am J Surg 1986;151:518-523.

12 Wood WJ, Rawlings M, Evans H, et al. Hepatocellular carcinoma: importance of histologic classification as a prognostic factor. Am J Surg 1988;155:663-666.

13 Okuda K. Natural history of hepatocellular carcinoma including fibrolamellar and hepato-cholangiocarcinoma variants. J Gastroenterol Hepatol 2002;17:401-405.

14 Pinna $\mathrm{AD}$, Iwatsuki S, Lee RG, et al. Treatment of fibrolamellar hepatoma with subtotal hepatectomy or transplantation. Hepatology 1997;26:877-883.

15 Everson GT, Trotter JF. Transplantation of the liver. In: Schiff ER, Sorrell MF, Maddrey WC (eds). Diseases of 
the Liver, 9th edn., Vol. 2. Lippincott, Williams and Wilkins: Philadelphia, 2003, pp 1585-1614.

16 Sherlock S, Dooley J (eds). Malignant liver tumors. In: Diseases of the Liver and Biliary System, 11th edn. Blackwell Science: Malden, MA, 2003, pp 537-562.

17 Rosai J. Rosai and Ackerman's Surgical Pathology. Mosby: Philadelphia, 9th edn., Vol. 1. 2004, pp 1001-1002.

18 Ferrell LD. Benign and malignant tumors of the liver. In: Odze RD, Goldblum JR, Crawford JM (eds). Surgical Pathology of the GI Tract, Liver, Biliary Tract and Pancreas. Saunders: Philadelphia, 2004, pp 1012.

19 Nagorney DM, Adson MA, Weiland LH, et al. Fibrolamellar hepatoma. Am J Surg 1985;149:113-119.

20 Haas JE, Muczynski KA, Krailo M, et al. Histopathology and prognosis in childhood hepatoblastoma and hepatocarcinoma. Cancer 1989;64:1082-1095.

21 Katzenstein HM, Krailo MD, Malogolowkin MH, et al. Fibrolamellar hepatocellular carcinoma in children and adolescents. Cancer 2003;97:2006-2012.

22 Hamilton SR, Aaltonen LA (eds). Tumors of the liver and intrahepatic bile ducts. In: Pathology and Genetics of the Digestive System. IARC Press: Lyon, 2000, pp 159-172.

23 Chedid A, Ryan LM, Dayal Y, et al. Morphology and other prognostic factors of hepatocellular carcinoma. Arch Pathol Lab Med 1999;123:524-528.

24 Quaglia A, Bhattacharjya S, Dhillon AP. Limitations of the histopathological diagnosis and prognostic assessment of hepatocellular carcinoma. Histopathology 2001;38:167-174.

25 Monto A, Wright TL. The epidemiology and prevention of hepatocellular carcinoma. Semin Oncol 2001; 28:441-449.

26 Vecchio FM, Federico F, Dina MA. Copper and hepatocellular carcinoma. Digestion 1986;35:109-114.
27 Lefkowitch JH, Muschel R, Price JB, et al. Copper and copper-binding protein in fibrolamellar liver cell carcinoma. Cancer 1983;51:97-100.

28 Guigui B, Mavier P, Lescs MC, et al. Copper and copper-binding protein in liver tumors. Cancer 1988; 61:1155-1158.

29 Garcia de Davila MT, Gonzalez-Crussi F, Mangkornkanok M. Fibrolamellar carcinoma of the liver in a child: ultrastructural and immunohistologic aspects. Pediatr Pathol 1987;7:319-331.

30 Wang JH, Dhillon AP, Sankey EA, et al. 'Neuroendocrine' differentiation in primary neoplasms of the liver. J Pathol 1991;163:61-67.

31 Caballero T, Aneiros J, Lopez-Caballero J, et al. Fibrolamellar hepatocellular carcinoma. An immunohistochemical and ultrastructural study. Histopathology 1985;9:445-456.

32 Zhao M, Laissue JA, Zimmermann A. 'Neuroendocrine' differentiation in hepatocellular carcinomas (HCCs): immunohistochemical reactivity is related to distinct tumor cell types, but not to tumor grade. Histol Histopathol 1993;8:617-626.

33 Huang XF, Wang CM, Dai XW, et al. Expressions of chromogranin A and cathepsin D in human primary hepatocellular carcinoma. World J Gastroenterol 2000; 6:693-698.

34 Orsatti G, Greenberg PD, Rolfes DB, et al. DNA ploidy of fibrolamellar hepatocellular carcinoma by image analysis. Hum Pathol 1994;25:936-939.

35 Wilkens L, Bredt M, Flemming P, et al. Cytogenetic aberrations in primary and recurrent fibrolamellar hepatocellular carcinoma detected by comparative genomic hybridization. Am J Clin Pathol 2000;114: 867-874

36 Okada K, Kim YI, Nakashima K, et al. Fibrolamellar hepatocellular carcinoma coexistent with a hepatocellular carcinoma of common type: report of a case. Surg Today 1993;23:626-631. 